\title{
Temperature Dependence of Viscoelasticity of Polycaprolactone Critical Gels
}

\author{
Akihiro Izuka and H. Henning Winter* \\ Chemical Engineering Department, University of Massachusetts, \\ Amherst, Massachusetts 01003
}

Takeji Hashimoto

Department of Polymer Chemistry, Faculty of Engineering, Kyoto University, Kyoto 606, Japan

Received March 9, 1994; Revised Manuscript Received August 15, $1994^{\circ}$

\begin{abstract}
A systematic procedure was developed for determining the temperature dependence of critical gel properties. The polymer system was a poly (e-caprolactone) diol (PCL) end-linked with a three-functional isocyanate. Samples varied in prepolymer molecular weight and stoichiometric ratio. Time-temperature superposition was found to apply to the self-similar relaxation modulus $G(t)=S t^{-n}$. A prerequisite of timetemperature superposition is that the relaxation exponent $n$ is independent of temperature. As a consequence, critical gels have the unique property that their loss tangent is independent of temperature. This was confirmed experimentally. The gel stiffness $S(T)=S\left(T_{0}\right) a_{T}{ }^{n} b_{T}$ shifts with an Arrhenius type temperature dependence. The temperature shift factors for the PCL critical gels seem to be identical with those for the viscosity of the difunctional prepolymer $\left(\eta_{0}\right)$ and the modulus of the fully cross-linked polymer $\left(G_{\theta}\right)$. This suggests that an experimental relationship of $S=G_{\mathrm{e}}(T)\left[\eta_{0}(T) / G_{\mathrm{e}}(T)\right]^{n}$ is valid for our polymers at the experimental temperatures between 90 and $130^{\circ} \mathrm{C}$.
\end{abstract}

\section{Introduction}

Temperature affects chemical gelation experiments in two major ways. High temperature accelerates the crosslinking reaction and thus speeds up the gelation process. It also accelerates relaxation processes so that the characteristic time scales of rheology become reduced. For analyzing the temperature dependence of gelation, we will use the rheology at the gel point as reference.

Cross-linking polymers undergo a transition from liquid (sol) to solid (gel) when the extent of cross-linking ( $p$ ) reaches a critical value at the gel point (GP). The leading molecular cluster and the corresponding longest relaxation time diverge to infinity for the ideal material exactly at GP, the critical gel (CG). The critical gel characteristically relaxes with a continuous power-law relaxation time spectrum ( $\mathrm{CW}$ spectrum $)^{1,2}$

$$
H(\lambda)=H_{0}\left(\lambda / \lambda_{0}\right)^{-n} \quad \lambda_{0}<\lambda<\infty
$$

which results in a relaxation modulus

$$
G(t)=S t^{-n} \quad \lambda_{0}<t<\infty
$$

The gel stiffness, $S=\lambda_{0}{ }^{n} H_{0} \Gamma(n)$, and relaxation exponent $(n)$ are the material parameters which completely characterize the linear viscoelastic behavior. The shortest time of the power-law behavior $\lambda_{0}$ marks the crossover to the glass transition or, for very high molecular weight precursors, to entanglement relaxation.

The same scaling behavior also governs dynamic mechanical experiments where the storage modulus $\left(G^{\prime}\right)$ and the loss modulus $\left(G^{\prime \prime}\right)$ at GP are related as

$$
G^{\prime}=G^{\prime \prime} / \tan \delta=S \omega^{n} \Gamma(1-n) \cos \delta \quad 0<\omega<1 / \lambda_{0}
$$

The phase angle between stress and strain $(\delta)$ is independent of frequency $(\omega)$ but proportional to the relaxation 1994.

Abstract published in Advance ACS Abstracts, September 15, exponent $t^{3}$

$$
\delta=n \pi / 2
$$

Many efforts to clarify the origin of the $\mathrm{CW}$ spectrum have focused on $n$ values of $\mathrm{CGs}$ with various molecular structures (molecular weight effects, stoichiometry, and dilution $)^{1-8}$ and on the fractal nature of CGs. ${ }^{9-13}$ The value of $n$ varies over about the entire possible range between 0 and 1 , depending on molecular composition and crosslinking conditions of any specific systems. Scanlan et al. ${ }^{4}$ studied a wide range of poly(dimethylsiloxane) (PDMS) cross-linking systems of intermediate molecular weight and found a linear relationship between $\log S$ and $n$

$$
S=G_{0} \lambda_{0}^{n}
$$

$G_{0}$ and $\lambda_{0}$ are material constants which, for end-linking systems, depend on the prepolymer molecular weight. $G_{0}$ was close to the equilibrium modulus of the fully crosslinked material $\left(G_{e}\right)$, and the product $G_{0} \lambda_{0}$ had about the value of the zero shear viscosity of the prepolymer $\left(\eta_{0}\right)$.

Izuka et al. ${ }^{9}$ investigated this relationship in further detail. They end-linked poly(t-caprolactone) diol (PCL) with a three functional isocyanate and found that the characteristic modulus $\left(G_{0}\right)$ in eq 5 is equal to the modulus of the fully cross-linked network $\left(G_{\theta}\right)$ and that the characteristic time $\left(\lambda_{0}\right)$ depends on the viscosity of the precursor $\left(\eta_{0}\right)$

$$
\lambda_{0}=\eta_{0} / G_{e}
$$

These results allow prediction of the gel stiffness

$$
S=G_{e}\left(\eta_{0} / G_{\mathrm{e}}\right)^{n}
$$

provided that the exponent $n$ is known. For all these polymers, the value of $n$ was found to decay with molecular weight of the precursor molecules.

As far as the temperature dependence, there has been only one report $t^{2}$ in which time-temperature superposition 
Table 1. Determined Values of the Relaxation Exponent $n$, tan $\delta$, and the Gel Stiffness $S$ of PCL Critical Gels at $110^{\circ} \mathrm{C}$

\begin{tabular}{lllllllllrr}
\hline & PCL4 & PCL7 & PCL9 & PCL20 & PCL7 & PCL7 & PCL7 & PCL7 & PCL7 & PCL7 \\
\hline$r^{a}$ & 1.00 & 1.00 & 1.00 & 1.00 & 0.61 & 0.80 & 1.12 & 1.20 & 1.41 & 1.76 \\
$n$ & 0.83 & 0.61 & 0.38 & 0.31 & 0.67 & 0.64 & 0.59 & 0.57 & 0.52 & 0.34 \\
$\tan \delta$ & 3.65 & 1.40 & 0.69 & 0.52 & 1.73 & 1.57 & 1.35 & 1.26 & 1.08 & 0.59 \\
$S(\mathrm{~Pa} \mathrm{~s})$ & $1.5 \times 10$ & $3.6 \times 10^{2}$ & $9.5 \times 10^{3}$ & $4.7 \times 10^{4}$ & $8.8 \times 10$ & $2.2 \times 10^{2}$ & $5.6 \times 10^{2}$ & $1.1 \times 10^{3}$ & $1.8 \times 10^{3}$ & $1.9 \times 10^{4}$
\end{tabular}

a Stoichiometric ratio, $r$, is defined as the initial molar ratio of isocyanate group to hydroxy group, [NCO]/[OH] .

was shown over a wide temperature range $(-50$ to +180 ${ }^{\circ} \mathrm{C}$ ) for PDMS critical gels and the values of the shift factors were determined. It was found that the horizontal shift factor $\left(a_{\mathrm{T}}\right)$ follows an Arrhenius relation.

The extent of reaction, $p$, is the independent variable of the cross-linking process. It is defined as the actual number of cross-links divided by the maximum possible number of cross-links. Within a limited temperature range it seems to be a reasonable assumption that the temperature changes should not affect the reaction mechanism so that the evolution of network structure is the same at low and high temperatures. The only difference would be expected in the rate of reaction, $\delta p / \delta t$.

Here, we study the temperature dependent viscoelasticity of PCL critical gels and relate it to the temperature dependent viscosity of the prepolymer and the modulus of the fully cross-linked material.

\section{Experiment}

Materials. Four poly(t-caprolactone) diols (PCLs) (commercial polymers of Daicel Chemical) were end-linked with a three-functional cross-linker, IPDI-T1890 supplied by Huels AG (poly-IPDI). Details of the component characterization and sample preparation were described previously. ${ }^{9}$ Viscoelastic properties of the four systems under isothermal conditions at $110^{\circ} \mathrm{C}$ are summarized in Table 1 . All sets of $(S, n)$ values were found to satisfy the relation of eq 7. PCL2 was excluded from this specific study because of its very low $S$ value at high temperatures.

Rheometer. The dynamic mechanical experiments were performed in a Rheometrics RDS 7700 with concentric disk fixtures. The prepolymers required large disks, $d=50 \mathrm{~mm}$, while gel properties and the final modulus were measured with disks of $d=25 \mathrm{~mm}$. The gap setting was about $1 \mathrm{~mm}$.

Determination of Gel Point at Different Temperatures. The viscoelastic properties change gradually during gelation. We therefore assume that time-temperature superposition, e.g. Ferry, ${ }^{14}$ of the dynamic modulus is valid not only in the sol before or the gel beyond GP but also at the gel point itself

$$
\begin{aligned}
& G^{\prime}\left(T, a_{T, C^{\omega}} \omega\right)=\left(1 / b_{T, \mathrm{C}}\right) G^{\prime}\left(T_{0}, \omega_{0}\right) \\
& G^{\prime \prime}\left(T, a_{T, C} \omega\right)=\left(1 / b_{T, \mathrm{C}}\right) G^{\prime \prime}\left(T_{0}, \omega_{0}\right)
\end{aligned}
$$

where $a_{T, C}$ and $b_{T, C}$ are the horizontal and vertical shift factors of the critical gel (CG), respectively, and $T_{0}$ is a reference temperature. By substituting eqs 8 into eq 3 , the temperature dependence of $S$ is obtained as

$$
S(T)=\left(a_{T, \mathrm{C}}{ }^{n} / b_{T, \mathrm{C}}\right) S\left(T_{0}\right)
$$

Time-temperature superposition is only possible if the slope of the $G^{\prime}, G^{\prime \prime}$ data remains constant in the entire temperature range. This means that the relaxation exponent $(n)$ is independent of temperature and that $\tan \delta$ at GP is also independent of temperature. Self-similar materials are rather unusual in that respect. We do not know of any other materials which would have $\tan \delta$ independent of temperature and frequency.

According to eqs 3 and 4, GP can be detected as a time when $\tan \delta$ is independent of frequency. To obtain $\tan \delta$, two methods were widely used:

The stopped samples method ${ }^{1}$ requires quenching of the reaction at a discrete set of $p$ values around the gel point. After stable samples are successfully obtained, the temperature de- pendent properties can be directly measured by varying the experimental temperature. Unfortunately, this method is not applicable here since we do not know of a good method which would allow us to rapidly stop the cross-linking reaction of the PCL system.

The second method does not require stopping of the reaction. It involves simultaneous ${ }^{15}$ or consecutive $e^{4}$ frequency scans during the cross-linking. Each data point is practically taken on a different state of the sample since the reaction is progressing, $p(t)$. Interpolation gives the evolving properties near the gel point. This, among other things, identifies the gel time by showing the instant at which $\tan \delta$ is independent of frequency. One condition has to be specifically met in this procedure: the rate of cross-linking has to be slow enough so that the change of modulus during the taking of a single data point is negligible:

$$
(1 / g)(\partial g / \partial t)(2 \pi / \omega) \leq 0.1
$$

Here $g$ stands for the measured quantity $\left(G^{\prime}, G^{\prime \prime}, \eta\right.$, etc. $)$ and $(2 \pi / \omega)$ is the experimental time for a dynamic experiment at constant frequency $\omega$. On the right hand side, a $10 \%$ change is suggested as a tolerable limit. The criterion shows that the needed low-frequency measurements are not possible any more if samples undergo very rapid cross-linking at high temperatures.

This condition can be evaluated for all experimental data. It has direct implications for our experiment. At an intermediate temperature of $110^{\circ} \mathrm{C}$, the reaction rate is still slow enough to allow complete frequency scans which reveal the gel point at which $\tan \delta$ is independent of frequency. More importantly, the experiment at $110^{\circ} \mathrm{C}$ gives the value of the relaxation exponent according to eq 4 . This value holds for all temperatures without change as long as temperature does not affect the reaction mechanism. Frequency scans at other temperatures should give the same value of $n$ and can be used for confirming the accuracy of the measurement at $110^{\circ} \mathrm{C}$.

We chose single frequency experiments at $10 \mathrm{rad} / \mathrm{s}$ and a maximum strain amplitude $\gamma_{R}=0.05$ in order to get a sufficiently strong torque signal. The oscillatory shear measurements were performed in the rheometer with $25 \mathrm{~mm}$ diameter parallel plates. All samples were reacted isothermally at $90,100,110,120$, and $130^{\circ} \mathrm{C}$ under a dried air atmosphere. Above $130^{\circ} \mathrm{C}$, the reaction rate was too high to measure the evolution of dynamic modulus even at the highest frequency.

\section{Experimental Results and Analysis}

Determination of $\eta_{0}$ and $G_{e}$. The temperature dependence of the initial and final properties can be written as (e.g. Ferry ${ }^{14}$ )

$$
\begin{gathered}
\eta_{0}(T)=\left(a_{T, 0} / b_{T, 0}\right) \eta_{0}\left(T_{0}\right) \\
G_{\mathrm{e}}(T)=\left(1 / b_{T, 1}\right) G_{\mathrm{e}}\left(T_{0}\right)
\end{gathered}
$$

where $a_{T, 0}, b_{T, 0}$, and $b_{T, 1}$ are the respective horizontal and vertical shift factors. The dynamic viscosity of each prepolymer was measured at temperatures of $80,90,100$, $110,120,130$, and $140^{\circ} \mathrm{C}$ and a maximum strain amplitude $\gamma_{R}=0.10$. All samples reached their zero shear viscosity, $\eta_{0}$, at frequencies between 0.1 and $10 \mathrm{rad} / \mathrm{s}$. The zero shear viscosity $\left(\eta_{0}\right)$ of all the prepolymers shifted with an Arrhenius relation (Figure 1).

$$
a_{T, 0} / b_{T, 0}=\exp \left(\Delta H_{\mathrm{f}} / R\left(1 / T-1 / T_{0}\right)\right)
$$




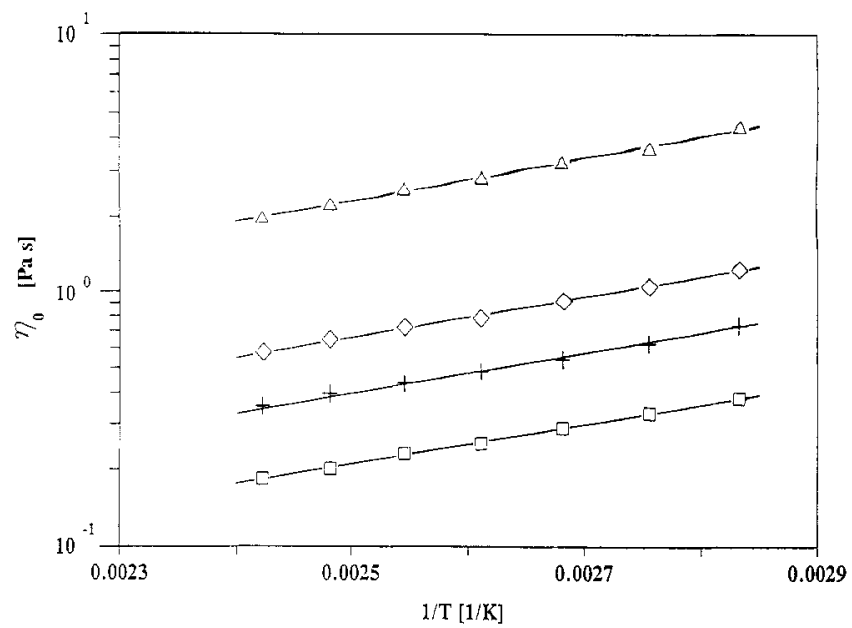

Figure 1. Arrhenius plots of the zero shear viscosities $\eta_{0}$ of PCL prepolymers including PCL4 (a), PCL7 $(+)$, PCL9 $(\diamond)$, and PCL20 ( $\Delta$ ).

where $\Delta H_{\mathrm{f}}$ is the activation energy of flow and $R$ is the ideal gas constant. Straight lines obtained by means of least-squares fits of the data points are almost parallel to each other, indicating that $\Delta H_{f}$ is almost independent of the prepolymer molecular weight. Its average value is calculated from the slopes as $\Delta H_{\mathrm{f}}=(1.83 \pm 0.09) \times 10^{2}$ $\mathrm{J} / \mathrm{mol}$.

The fully cross-linked samples were prepared from the same mixtures as the critical gels of Izuka et al. ${ }^{9}$ After curing in the rheometer at $130^{\circ} \mathrm{C}$ for $14 \mathrm{~h}$, the dynamic moduli did not change anymore. Dynamic experiments were performed at temperatures between 80 and $140^{\circ} \mathrm{C}$ with frequencies between 0.1 and $100 \mathrm{rad} / \mathrm{s}$ and $\mathrm{a}$ maximum strain amplitude $\lambda_{\mathrm{R}}=0.01$. The modulus $\left(G^{*}\right)$ values of the fully cross-linked samples were almost independent of frequency in the experimental temperature range. We adopted the $G^{*}$ value at $\omega=0.1 \mathrm{rad} / \mathrm{s}$ as $G_{\mathrm{e}}$ for further discussions.

In order to compare the result with classical rubber theory ${ }^{16}$

$$
G_{\mathrm{e}}=\left(\rho R / M_{\mathrm{c}}\right) T
$$

where $M_{\mathrm{c}}$ is the molecular weight between cross-linking points, the product of $G_{\theta}$ and the number average molecular weight of the precursors $\left(M_{\mathrm{n}}\right)$, which almost corresponds to $M_{c}$, is plotted against the absolute temperature for the stoichiometrically balanced systems (see Figure $2 a$ ). The linear proportionality of $G_{\mathrm{e}} M_{\mathrm{n}}$ with absolute temperature suggests that the change of density $\rho(T)$ is negligible in this temperature range. The $G_{e} M_{n}$ values are about the same for all molecular weights except for PCL20. The higher $G_{\mathrm{e}} M_{\mathrm{n}}$ value of PCL20 might be attributed to physical entanglements trapped in the chemically crosslinked network structure.

The modulus $G_{\mathrm{e}}$ depends on stoichiometry. For imbalanced systems, the meaning of $M_{\mathrm{c}}$ is not as clearly defined and it is more informative to plot $G_{\mathrm{e}}$ against temperature $T$; see Figure $2 \mathrm{~b} . G_{\mathrm{e}}$ is again proportional to $T$ which means that $\rho(T)$ does not significantly depend on temperature. Therefore, one can conclude that $b_{T, 1}$ is approximately described as

$$
b_{T, 1}=\rho T / \rho_{0} T_{0} \simeq T / T_{0}
$$

for all the systems.

Effect of Temperature on the Cross-Linking Reaction. The loss tangent, $\tan \delta$, decreases during isothermal cross-linking, as shown in Figure 3 for a stoichiometrically
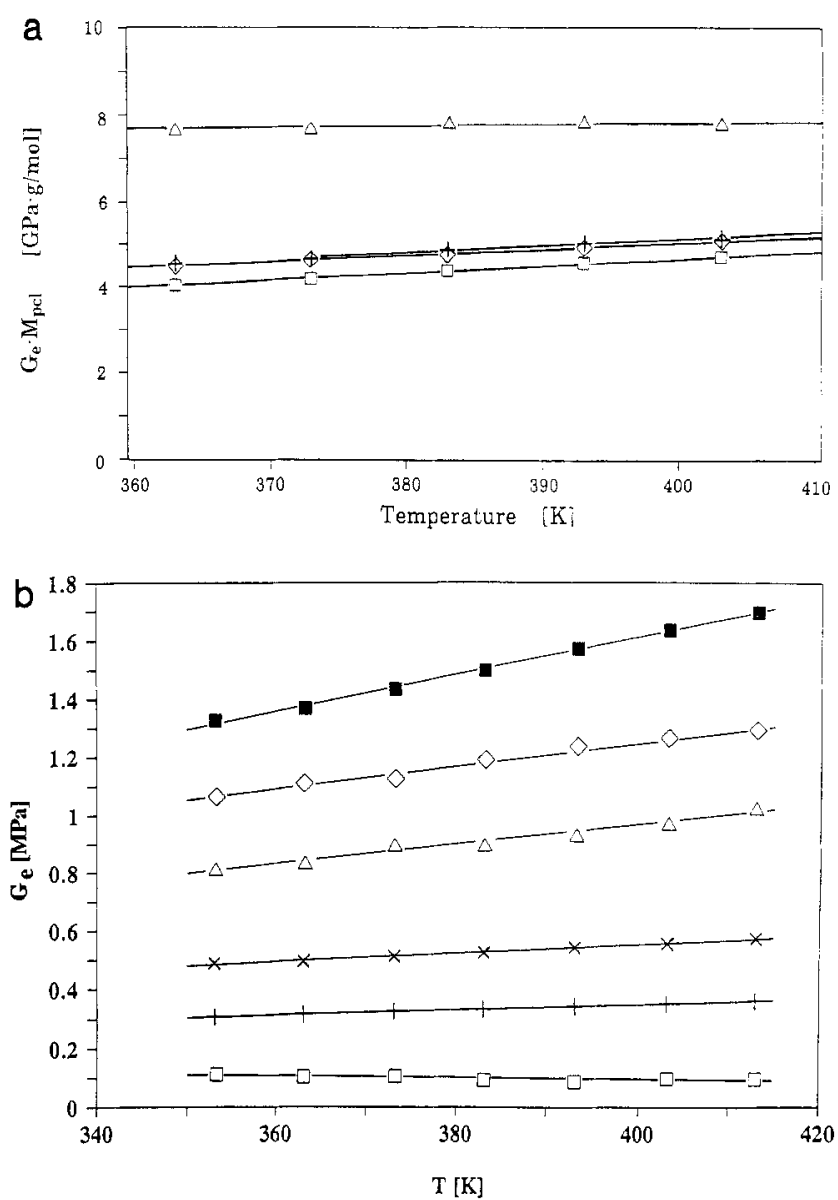

Figure 2. Equilibrium moduli $G_{0}$ of the fully cross-linked materials plotted against the absolute temperature. Figure $2 \mathrm{a}$ is for stoichiometrically balanced systems of PCL4 (ㅁ), PCL7 $(+)$, PCL9 $(\diamond)$ and PCL20 $(\Delta), G_{e}$ is multiplied by the molecular weight of the precursor which ideally determines the molecular weight between cross-links. Figure 2b is for PCL7 systems with stoichiometric ratios of $0.61(\square), 0.80(+), 1.12(\square), 1.20(\diamond), 1.41$ $(\Delta)$ and $1.76(X)$.

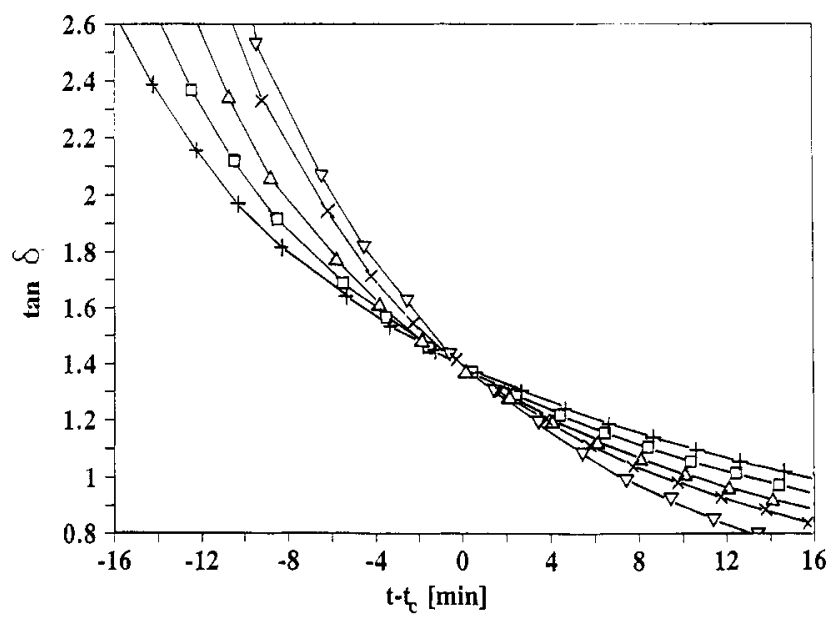

Figure 3. Evolution of $\tan \delta$ of a stoichiometrically balanced PCL7 system during isothermal cross-linking reaction at $110^{\circ} \mathrm{C}$ with a frequency of $1(\mathrm{rad} / \mathrm{s})(\nabla), 3.162(\times), 10(\Delta), 31.62(\square)$, and $100(+)$.

balanced PCL7 system at $110^{\circ} \mathrm{C}$. The instant at which $\tan \delta$ was independent of frequency determines the GP according to eq 3 . The PCL7 critical gel had values of tan $\delta=1.4$ and $n=0.61$. The corresponding evolution of the dynamic modulus ( $G^{\prime}$ and $G^{\prime \prime}$ ) as measured at a single frequency of $\omega=10 \mathrm{rad} / \mathrm{s}$ strongly depends on the temperature of the cross-linking process (see Figure 4). For each sets of curves, the observed GP is indicated as 


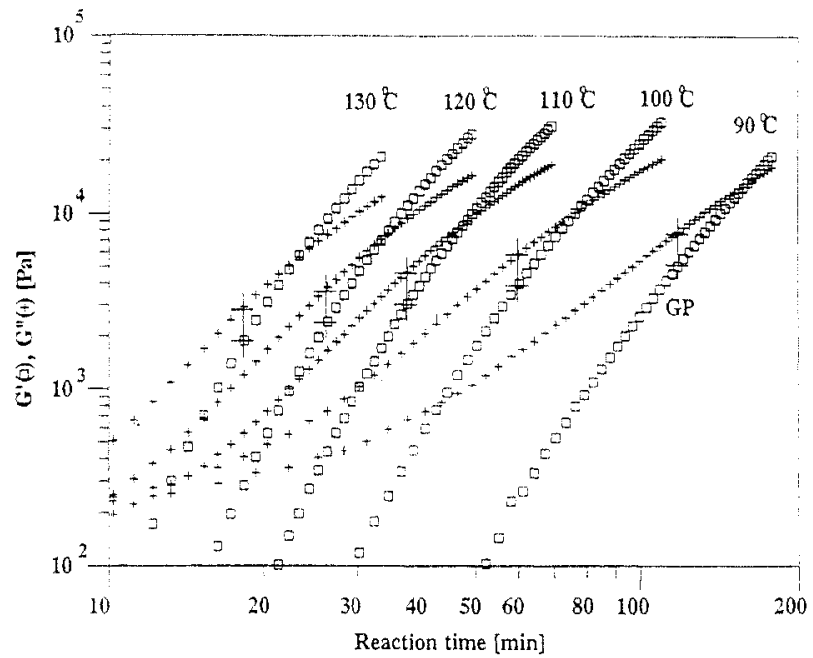

Figure 4. Evolution of the dynamic modulus of stoichiometrically balanced PCL4 (a), PCL7 (b), PCL9 (c), and PCL20 (d) during isothermal cross-linking reaction at $90,100,110,120$, and $130^{\circ} \mathrm{C}$ measured at a constant frequency of $10 \mathrm{rad} / \mathrm{s}$. The crosses mark the gel point.

the instant at which $\tan \delta$ is equal to the value at the gel point $(\tan \delta=1.4$ for PCL 7). The GP values of the dynamic modulus $\left(G^{\prime}{ }_{c}\right.$ and $\left.G^{\prime \prime}{ }_{c}\right)$ decreased slightly with increasing temperature. Similar results were obtained for all the other systems and GPs were determined on the basis of the corresponding $n$ values listed in Table 1 .

The gel point is reached much earlier at a high temperature due to the increased rate of reaction $\partial p / \partial t$. The $G^{\prime}, G^{\prime \prime}$ curves could be compared directly if they were plotted against the conversion $p$ instead of the reaction time. Such a comparison is possible if one adjusts the time scale of the evolving rheology data. This is done most simply by assuming that, in the vicinity of GP, the relative modulus change

$$
R^{*}=\left(1 / G^{*}\right)\left(\partial G^{*} / \partial t\right)=\left(1 / G^{*}\right)\left(\partial G^{*} / \partial p\right)(\partial p / \partial t)
$$

can be decomposed into a rate of change of property $G^{*}$ as a function of conversion

$$
\left(1 / G^{*}\right)\left(\partial G^{*} / \partial p\right) \neq f(T)
$$

which is nearly independent of temperature, and a rate of reaction $\partial p / \partial t$ which contains the temperature dependence.

This still does not allow us to express the temperature in absolute terms. However, the relative rate of change

$$
R^{*}(T) / R^{*}\left(T_{0}\right) \simeq(\partial p / \partial t)_{T} /(\partial p / \partial t)_{T_{0}}
$$

gives us the effect of the temperature $T$ as compared to cross-linking at temperature $T_{0}$. All PCL samples have the same temperature dependence at GP; see Figure 5. The straight line indicates an Arrhenius type

$$
R_{\mathrm{c}}^{*}(T) / R_{\mathrm{c}}^{*}\left(T_{0}\right)=\exp \left\{\alpha\left(1 / T-1 / T_{0}\right)\right\}
$$

where $\alpha$ is determined to be $-3.27 \times 10^{3}(\mathrm{~K})$. With this function, the temperature dependence can be expressed as well as with $p(t, T)$ data (which are not available to us).

The rate of modulus change has been measured (slope in Figures 4) and can now be used to adjust the time scales in Figures 4 to obtain master curves. After adjusting the time scale, $G^{\prime}, G^{\prime \prime}$ curves have a shape which is independent of temperature. This can be observed more clearly by normalizing the vertical axis with a factor of $G_{c}^{\prime}(T) / G^{\prime} c^{-}$

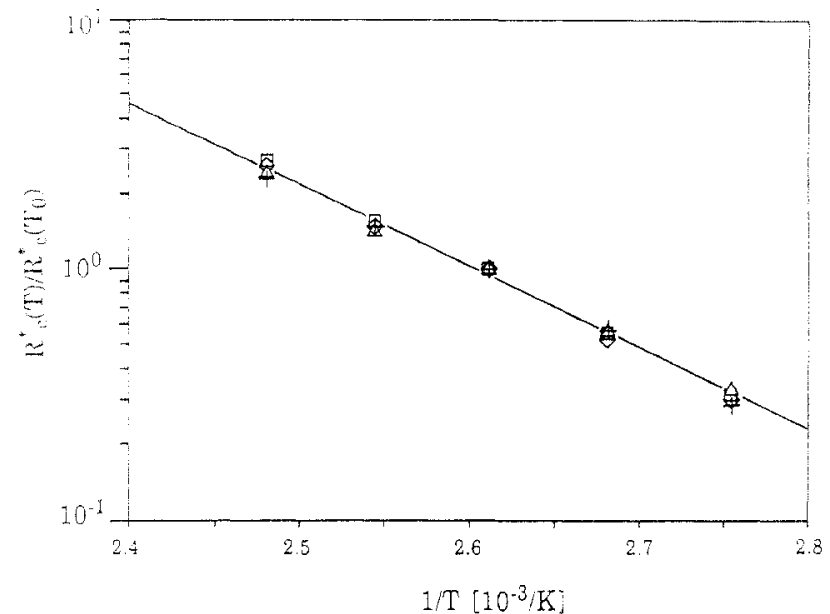

Figure 5. Relative rates of modulus change $R^{*}{ }_{c}(T) / R^{*}{ }_{c}\left(T_{0}\right)$ as a function of temperature for stoichiometrically balanced systems of PCL4 (a), PCL7 (t), PCL9 ( $)$ ), and PCL20 ( $\Delta) . T_{0}=110$ ${ }^{\circ} \mathrm{C}$ is the reference temperature. Subscript $\mathrm{c}$ indicates that the rates were evaluated at GP.

$\left(T_{0}\right)$. The evolution curves are almost superposed onto each other, especially in the vicinity of GP; see Figure 6. This suggests that the molecular structure of the crosslinking PCL is identical at different temperatures if we probe it rheologically at the same extent of reaction.

Determination of Temperature Dependent $S$. Since the dynamic modulus of $C G$ is a linear function of frequency with slope $n$ according to eq 3 , we cannot distinguish the contribution of $a_{T, 1}$ and $b_{T, 1}$ to the temperature dependence even if we know $G^{\prime}$ and $G^{\prime \prime}{ }_{\mathrm{c}}$ from measurements over a wide range of frequencies at different temperatures. However, the ratio of $G_{c}^{\prime}(T) / G_{c}^{\prime}\left(T_{0}\right)$ or $G^{\prime \prime}{ }_{c^{-}}$ $(T) / G^{\prime \prime}{ }_{\mathrm{c}}\left(T_{0}\right)$ at a constant frequency becomes equal to $a_{T, \mathrm{C}^{n} /}$ $b_{T, \mathrm{C}}$, which corresponds to the temperature shift factor of $S, S(T) / S\left(T_{0}\right)$, according to eq 9 :

$$
\begin{aligned}
G^{\prime}(T) / G^{\prime}{ }_{c}\left(T_{0}\right) & =G^{\prime \prime}{ }_{c}(T) / G^{\prime \prime}{ }_{c}\left(T_{0}\right) \\
& =a_{T, C}{ }^{n} / b_{T, C} \\
& =S(T) / S\left(T_{0}\right)
\end{aligned}
$$

For this reason, a single-frequency experiment is sufficient for determination of $S(T) / S\left(T_{0}\right)$. The average $G_{\mathrm{c}^{-}}$ $(T) / G^{\prime}{ }_{c}\left(T_{0}\right)$ and $G^{\prime \prime}{ }_{c}(T) / G^{\prime \prime}{ }_{c}\left(T_{0}\right)$ are plotted against the reciprocal of the absolute temperature in Figure 7. The steepest slope occurred when decreasing the absolute value of $S$ or increasing $n$, according to eq 5 . They seem to follow an Arrhenius relation.

\section{Discussion}

The experiments have shown that the relaxation exponent of a critical gel does not depend on the temperature at which the cross-linking has occurred. We conclude from that observation that, within the experimental $T$ range, the type of structural evolution is independent of temperature. It just occurs faster when the temperature is increased. This observation has significant implication for the experiments (or will complicate experiments where it does not hold): The relaxation exponent $(n)$ has to be measured only once, for instance by using the tan $\delta$ method. ${ }^{15}$ This should be done at a temperature which allows the easiest experiments. For all further samples (of the same composition), the gel point can simply be determined by "fitting" on one frequency and waiting until the ratio $G^{\prime \prime} / G^{\prime}$ passes through the known value of $\tan (n \pi / 2)$. This method is especially useful at 
a

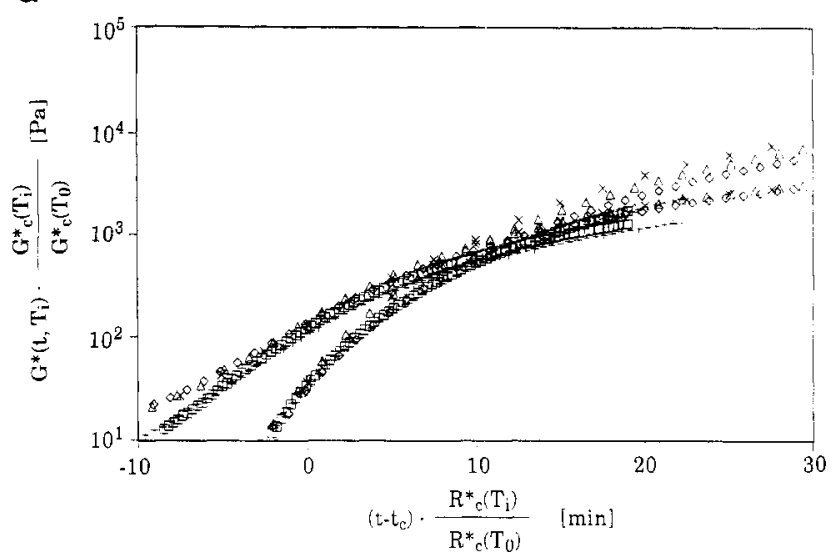

b

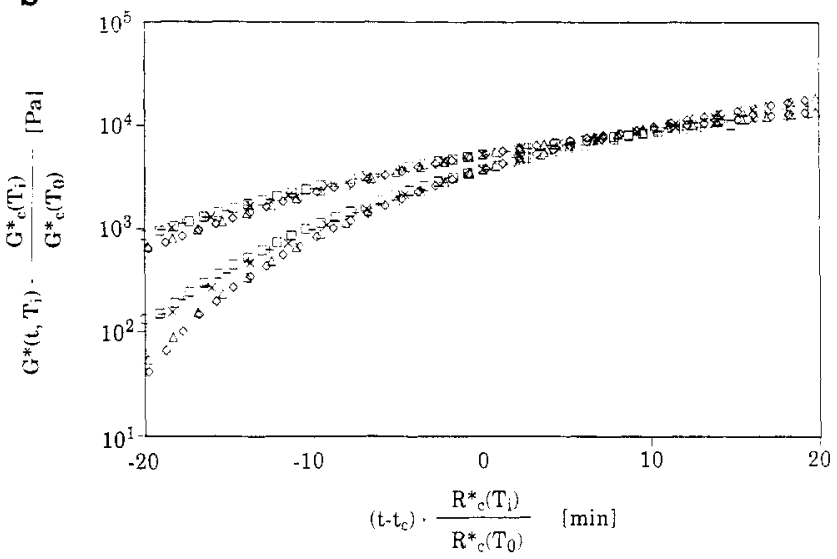

C

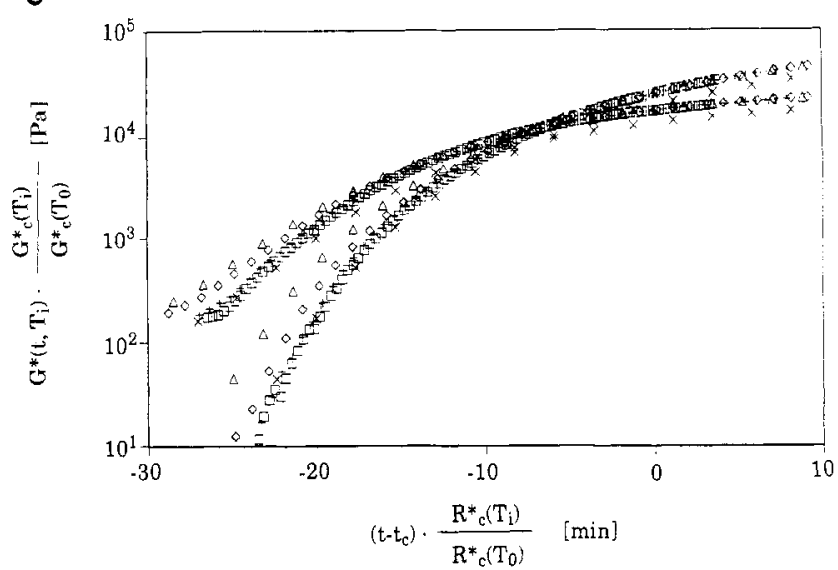

d

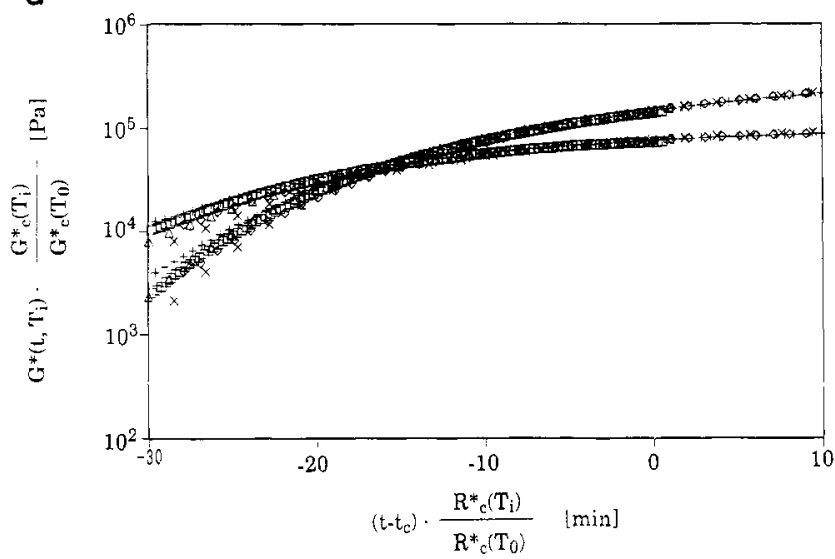

Figure 6. Normalized dynamic modulus by $G_{c}(T) / G_{c}\left(T_{0}\right)$ of stoichiometrically balanced systems of PCL4 (a), PCL7 (b), PCL 9 (c), and PCL20 (d) during isothermal cross-linking reaction at $90(\mathrm{D}), 100(+), 110(\diamond), 120(\Delta)$, and $130^{\circ} \mathrm{C}(\times)$, measured at a frequency of $10 \mathrm{rad} / \mathrm{s}$, plotted against $\left(t t_{\mathrm{c}}\right) R^{*}(T) / R^{*}\left(T_{0}\right)$. A reference temperature is $T_{0}=110^{\circ} \mathrm{C}$.

high temperature and, hence, high reaction rates $(\partial p / \partial t)$ where only limited experimental time is available. The data from this procedure, Figure 7, are consistent for our system.

The experiments give a composite shift factor of $S(T)$ (see eq 20); but they do not separate the information into a horizontal and vertical shift. However, the shift factors depend on the chemical details of the molecular chains, i.e. a chain friction factor (Ferry ${ }^{14}$ ), and can be assumed to be generic for the polymeric system. For an initial analysis we evaluate the most simple proposal which assumes that the shift factors of prepolymers $\left(a_{T, 0}, b_{T, 0}\right)$ and fully cross-linked polymer $\left(b_{T, 1}\right)$ are identical with the shift factors of $\mathrm{CG}$ as given in eq 11. In this case, $a_{T, \mathrm{C}}$ and $b_{T, \mathrm{C}}$ are represented by

$$
\begin{gathered}
a_{T}=a_{T, \mathrm{C}}=\left(a_{T, 0} / b_{T, 0}\right) b_{T, 1} \\
b_{T}=b_{T, \mathrm{C}}=b_{T, 1}
\end{gathered}
$$

The experimentally determined values of $a_{T, 0} / b_{T, 0}$ and $b_{T, 1}$, substituted into eq 9 , predict the temperature dependence of $S$

$$
S(T)=\left(a_{T, 0}\left(b_{T, 0}\right)^{n}\left(b_{T, 1}\right)^{n-1} S\left(T_{0}\right)\right.
$$

This prediction has been calculated for all systems and is shown in Figure 7 as well. $(\log S)$ appears to be almost linear in $1 / T$ because the contribution of $b_{\mathrm{T}, 1}$ to $S(T) /$ $S\left(T_{0}\right)$ is negligible compared with the contribution of $a_{T, 1} /$ $b_{T, 1}$, which follows an Arrhenius equation. The lines show good agreement with the experimental values, and it is appropriate to assume that the shift factors of $C G$ are well represented with the shift factors of prepolymer and fully cross-linked polymer.

It would be interesting to repeat the above experiments with a system of higher glass transition temperature $\left(T_{\mathrm{g}}\right)$. There, the temperature effects of gelation would relate to $T_{\mathrm{g}}$ and might have a universal WLF form. ${ }^{17}$ This WLF format was explained in terms of free volume change and suggests that the horizontal shift factor depends only on $T_{\mathrm{g}}$. Although $T_{\mathrm{g}}$ increases with the extent of cross-linking, the increase is not significant as long as the cross-linking density does not exceed a critical value. ${ }^{18}$ Since the increase of $T_{\mathrm{g}}$ due to cross-linking was small even after the completion of the cross-linking (Izuka et al. ${ }^{9}$ ), the CGs of the PCL system are expected to have almost the same $T_{\mathrm{g}}$ as the prepolymer, indicating that cross-linking would not noticeably change the horizontal shift factor.

On the other hand, the vertical shift is related to thermal expansion as described in eq 15 so that it might be affected by cross-linking. However, the vertical shift factor was found to be almost equal to 1 for our samples. This means that the vertical shift due to cross-linking is very small. If we assume that the change of $\rho(T)$ is negligible, as in the fully cross-linked samples, $b_{T}=T / T_{0}$ is calculated as 0.922 and 1.05 at the lowest and the highest experiment temperatures, respectively. The above discussion supports the proposed model.

Among all the parameters in eq 22, only $n$ was found to depend on the prepolymer molecular weight and the stoichiometric ratio. This implies that the effects of the prepolymer molecular weight and the stoichiometric ratio 
a
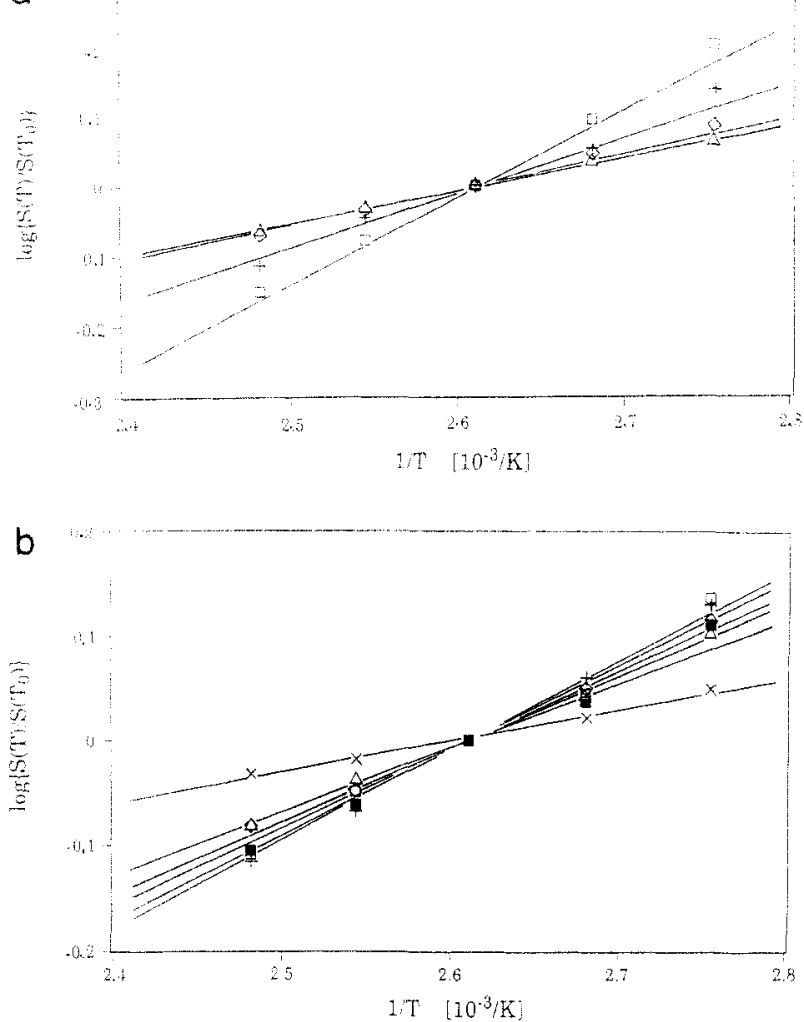

Figure 7. Arrhenius plots of $S(T) / S\left(T_{0}\right)$ of stoichiometrically balanced systems (a) of PCL4 (a), PCL7 (+), PCL9 ( $\diamond)$, and PCL20 $(\Delta)$ and PCL7 systems $(b)$ with stoichiometric ratios of $0.61(\square), 0.80(+), 1.12(\square), 1.20(\diamond), 1.41(\Delta)$, and $1.76(\times)$. Solid lines are calculated on the basis of the equation $S(T) / S\left(T_{0}\right)=$ $\left(a_{\mathrm{T}, 0} / b_{\mathrm{T}, 0}\right)^{n}\left(b_{\mathrm{T}, 1}\right)^{n-1}$

enter through only $n$. Equation 22 also suggests that $S(T) /$ $S\left(T_{0}\right)$ in the limit of the hardest gel $(n \rightarrow 0)$ and the softest gel $(n \rightarrow 1)$ should be equal to $b_{T, 1}$ and $a_{T, 0} / b_{T, 0}$, respectively.

We now can return to the proposal of Scanlan et al. ${ }^{4}$ and Izuka et al..$^{9}$ to express $S$ in terms of the modulus of the fully cross-linked system $\left(G_{e}\right)$ and the viscosity of the prepolymer $\left(\eta_{0}\right)$. This relation had been shown to be valid at temperature $T_{0}=110^{\circ} \mathrm{C}$. The above experiments suggest that it can be extended to other temperatures $T$ by including the temperature dependence of $\eta_{0}(T)$ and $G_{\mathrm{e}}(T)$

$$
S(T)=G_{e}(T)\left(\eta_{0}(T) / G_{e}(T)\right)^{n}
$$

One of the limitations of eq 22 might come from prepolymer molecular weight. If we start with a very low molecular weight prepolymer, the increase of $T_{\mathrm{g}}$ due to cross-linking would be significant and we might not see such good agreement. An example is end-linking poly(propylene oxide) with a low molecular weight of prepolymer, in which $T_{\mathrm{g}}$ significantly increases during crosslinking. Such systems do not follow the relationship of eq $7,5,6$ It is quite expected in such a system, since even if it exhibits the relationship of eq 7 at a particular temperature, a change of shift factors during cross-linking causes failure of eq 7 at any other temperature.

\section{Conclusions}

The temperature dependence of the critical gel rheological properties was successfully determined by means of oscillatory shear measurements for an end-linking poly( $\epsilon$-caprolactone) system. While the relaxation exponent $(n)$ does not depend on temperature, the temperature dependence of $S(T)$ can be predicted by the horizontal shift factor $\left(a_{T, 0}\right)$ and the vertical shift factor $\left(b_{T, 0}\right)$ of the initial viscosity $\left(\eta_{0}\right)$ of the prepolymer and the vertical shift factor $\left(b_{T, 1}\right)$ of the final modulus $\left(G_{e}\right)$ of the fully cross-linked polymers. The resulting equation of $S(T)=$ $\left(a_{T, 0} / b_{T, 0}\right)^{n}\left(b_{T, 1}\right)^{n-1} S\left(T_{0}\right)$, based on the assumption that the shift factors of prepolymer and fully cross-linked polymers are identical with the shift factor of $\mathrm{CG}$, has been confirmed experimentally for our system.

Temperature dependence of the reaction rate may be expressed with the rate of change of moduli $R^{*}(T)$ at the gel point. This allows shifting of the data onto a single reference temperature $T_{0}$.

This result suggests that $S(T) / S\left(T_{0}\right)$ depends only on the relaxation exponent $(n)$ and that an experimental relationship of $S=G_{\mathrm{e}}\left(\eta_{0} / G_{\mathrm{e}}\right)^{n}$ can be observed at the experimental temperatures.

The density of the polymer sample changes only very little with temperature. This simplifies the data analysis since the vertical shift is small because of this reason. It would be interesting to repeat the above experiments with a polymer of large expansion coefficient.

Acknowledgment. We gratefully acknowledge Daicel Chemical Industries, Ltd., for financial support and providing samples. We also thank Huels AG for samples of the cross-linker.

\section{References and Notes}

(1) Chambon, F.; Winter, H. H. Polym. Bull. 1985, 13, 499.

(2) Winter, H. H.; Chambon, F. J. Rheol. 1986, 30, 367.

(3) Chambon, F; Winter, H. H. J. Rheol. 1987, 31, 683.

(4) Scanlan, J. C.; Winter, H. H. Macromolecules 1991, 24, 47.

(5) Chambon, F.; Petrovic, Z. S.; MacKnight, W. J.; Winter, H. H. Macromolecules 1986, 19, 2146.

(6) Winter, H. H.; Morganelli, P.; Chambon, F. Macromolecules $1988,21,532$.

(7) Rubinstein, M.; Colby, R. H.; Gilmor, J. R. Polym. Prepr. (Am. Chem. Soc., Div. Polym. Chem.) 1989, 30, 81.

(8) Muller, R.; Gerard, E.; Dugand, P.; Rempp, P.; Gnanou, Y. Macromolecules 1991, 24, 1321.

(9) Izuka, A.; Winter, H. H.; Hashimoto, T. Macromolecules 1992 $25,2422$.

(10) Muthukumar, M. J. Chem. Phys. 1985, 83, 3161.

(11) Durand, D.; Delsanti, M.; Adam, M.; Luck, J. M. Europhys. Lett. 1987, 3, 97.

(12) Martin, J. E.; Adolf, D.; Wilcoxon, J. P. Phys. Rev. Lett. 1988, 61,2620 .

(13) Muthukumar, M. Macromolecules 1989, 22, 4656.

(14) Ferry, J. D. Viscoelastic Properties of Polymers, 3rd ed.; John Wiley and Sons: New York, 1980.

(15) Holly, E. E.; Venkataraman, S. K.; Chambon, F.; Winter, H. H., J. Non-Newt. Fluid Mechanics 1988, 27, 17.

(16) Treloar, L. R. G. The Physics of Rubber Elasticity; Clarendon Press: London, 1975.

(17) Williams, M. L.; Landell, R. F.; Ferry, J. D. J. Am. Chem. Soc. $1955,77,3701$.

(18) Fox, T. G.; Loshrek, S. J. Polym. Sci. 1955, 15, 371 